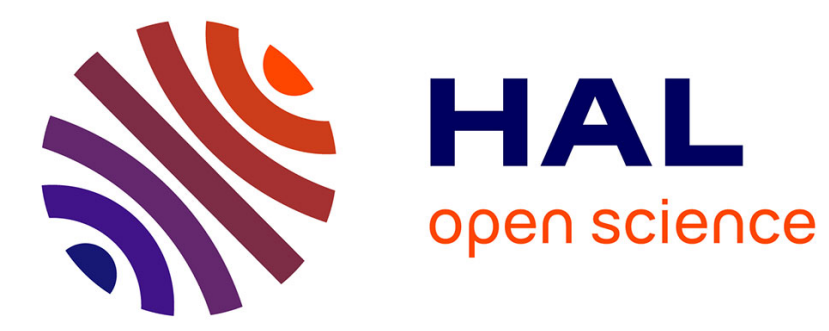

\title{
Microsatellite-based parentage analysis reveals non-ideal free distribution in a parasitoid population
}

Cédric Tentelier, Thomas T. Guillemaud, S. Ferry, Xavier Fauvergue

\section{To cite this version:}

Cédric Tentelier, Thomas T. Guillemaud, S. Ferry, Xavier Fauvergue. Microsatellite-based parentage analysis reveals non-ideal free distribution in a parasitoid population. Molecular Ecology, 2008, 17 (9), pp.2300-2309. 10.1111/j.1365-294X.2008.03743.x . hal-02658687

\section{HAL Id: hal-02658687 https://hal.inrae.fr/hal-02658687}

Submitted on 15 Sep 2020

HAL is a multi-disciplinary open access archive for the deposit and dissemination of scientific research documents, whether they are published or not. The documents may come from teaching and research institutions in France or abroad, or from public or private research centers.
L'archive ouverte pluridisciplinaire HAL, est destinée au dépôt et à la diffusion de documents scientifiques de niveau recherche, publiés ou non, émanant des établissements d'enseignement et de recherche français ou étrangers, des laboratoires publics ou privés. 


\title{
Microsatellite-based parentage analysis reveals non-ideal free distribution in a parasitoid population
}

\author{
CÉDRIC TENTELIER, THOMAS GUILLEMAUD, SARAH FERRY and XAVIER FAUVERGUE \\ UMR INRA, CNRS, Université de Nice-Sophia Antipolis 1301, Interactions Biotiques en Santé Végétale, BP 167 400, Routes des \\ Chappes, 06903 Sophia Antipolis cedex, France
}

\begin{abstract}
Habitat selection by dispersers is the focus of much theoretical models, most of which are based on the assumption of negative density dependence. The archetype of these models is the ideal free distribution, characterized by an evolutionary stable state where more competitors aggregate in better habitats, so that the fitness benefit of resource abundance is equally offset by the cost of competition in all habitats. In this study, we used parentage analysis on microsatellite genotypes to test the ideal free distribution in a natural population of aphid parasitoids. Parentage analysis was conducted on parasitoids emerging from aphid colonies. We inferred the number of foundress females which had reproduced in each colony, as well as the number of offspring for each foundress. As predicted by the ideal free distribution, the number of offspring per foundress per colony did not depend on the number of hosts per colony. However, contrary to ideal free distribution predictions, it was affected by the number of foundresses per colony. In surprising contrast with the basic assumption of negative density dependence, individual fitness increased with the number of foundresses. Moreover, parentage analysis revealed a very low number of offspring per foundress per colony (mean $=1.8$ ). This observed distribution questions the validity of classical models of habitat choice based on competition. Indeed, our results provide a new illustration reinforcing a growing body of theory and data on positive density dependence. Our results also suggest that the avoidance of hyperparasitism and predation, although generally neglected, may shape the distribution of parasitoids in the field.
\end{abstract}

Keywords: Aphis nerii, density-dependence, dispersal, Lysiphlebus testaceipes, relatedness, secondary parasite

\section{Introduction}

Dispersal is a major ecological process, the study of which benefits tremendously from the development of genetic markers and the statistical analysis of genetic data. Ever since the 'allozymic era', Wright's F-statistics have been used to estimate the number of dispersing individuals per generation. When regressed against Euclidean distance between populations, Wright's $F_{\mathrm{ST}}$ also quantifies isolation by distance, and allows the distance travelled by genes at every generation, that is, dispersal range, to be estimated

Correspondence: Cédric Tentelier, Université de Pau et des Pays de l'Adour, UMR-INRA ECOBIOP, Allée du parc Montaury, 64600 Anglet, France. Fax: +33 559574429;

E-mail: cedric.tentelier@univ-pau.fr
(Rousset 1997). With the development of highly variable markers, genetic methods entered the toolkit of students of dispersal at the individual level. For instance, when coupled with spatial information, assignment of individuals to the populations from where they originate allows identifying dispersers and determining individual dispersal distance (e.g. Berry et al. 2004). Then one can correlate the probability or distance of dispersal to other traits to identify internal or environmental determinants of dispersal (e.g. Castric \& Bernatchez 2004).

Habitat selection is a crucial stage of dispersal to which the analysis of genetic data and more specifically, parentage analysis, could be fruitfully applied. Relatedness estimates have already been used to show the role of both kin facilitation and avoidance of kin competition in the distribution of several organisms (e.g. prairie vole, Sera \& Gaines 1994; 
African wild dog, Girman et al. 1997; brown trout, Carlsson et al. 2004; black bear, Moyer et al. 2006). Yet, the usefulness of parentage analysis extends well beyond the special case of kin-biased habitat choice. Indeed, the choice of an individual for specific breeding habitats translates in the distribution of its offspring. Thus, by performing parentage analysis on individuals sampled in known habitats before they disperse, one can infer the decisions made by their parents at the time they had to choose their breeding sites. Such a method both gives an indirect cue of the presence of an individual (its genes, left in its offspring) and quantifies its reproductive output, thereby allowing to estimate the adaptive value of habitat selection.

A leading paradigm in habitat choice theory is the ideal free distribution (IFD; Fretwell \& Lucas 1970). It predicts that when individuals with equal competitive value exploit a patchily distributed resource and when each individual decides to exploit the patch that maximizes its fitness, the population reaches an evolutionarily stable state where the negative effect of competitors' number balances the positive effect of resource quantity in the patch. The IFD predicts that at equilibrium, (i) the individual gain of fitness is the same across all patches of the habitat (with the functional responses which are generally assumed, this means that gain is independent of the number of competitors and quantity of resources in the patches; Fauvergue et al. 2006), and (ii) the number of individuals exploiting a patch increases with the quantity of resources in the patch. The difficulty to obtain a reliable indicator of individual fitness in the field has constrained empirical tests of the IFD either to test solely prediction (ii) in wild populations (Kennedy \& Gray 1993; but see Wahlstrom \& Kjellander 1995; Beckmann \& Berger 2003), or to transpose the IFD to foraging situations, where the proxy for fitness gain is the amount of food ingested by the foragers (Tregenza 1995; Cezilly \& Benhamou 1996). Parentage analysis circumvents this constraint because, as mentioned above, it allows to infer both the presence of an individual reproducing on a site and its offspring production, which is closely related to fitness. Using parentage analysis, the predictions of the IFD therefore become (i) clutch size is the same across all patches, and (ii) the number of clutches in a patch increases with the quantity of resource in the patch.

In this study, we use parentage analysis to study the distribution of a natural population of the aphid parasitoid Lysiphlebus testaceipes Cresson (Hymenoptera: Braconidae), taking the IFD as a null model. Like many other parasitoids, female L. testaceipes disperse between a number of host patches, distributing their offspring among several sites (breeding dispersal). Such a high rate of movement in conjunction with the minute size of adults $(c .2 \mathrm{~mm})$ precludes the test of the IFD in natural populations using direct individual observations, and makes parentage analysis a very valuable tool in such a study. Moreover, aphid parasitoids in general, and this species in particular, have several features that make them a suitable model for our experiment. First, their hosts are heterogeneously distributed in the habitat and form colonies bearing highly variable number of aphids (Rabasse \& Wyatt 1985). This ensures to test the predictions of the IFD on a wide range of patch profitabilities. Second, aphids inside which a parasitoid pupates become light brown and swollen (they are then referred to as mummies), and are therefore easily distinguishable from unparasitized aphids. This allows to sample only patches with parasitized hosts, and to monitor emergence from parasitized hosts only (see Methods section). Third, and more specific to L. testaceipes, females are able to estimate patch profitability and habitat-wide profitability, and adapt their patch residence time accordingly (Tentelier et al. 2005; Tentelier et al. 2006; Tentelier \& Fauvergue 2007). Such an adaptive flexibility theoretically allows convergence of the population towards the IFD (Bernstein et al. 1988). Fourth, and related to this convergence, L. testaceipes is the species on which Fauvergue et al. (2006) tested predictions of the IFD model of Bernstein et al. (1988). Their test was carried out by releasing parasitoids in a glasshouse containing 40 artificially initiated aphid patches. Comparison between their results and ours will point at differences in the ecological factors affecting parasitoid distribution in a simplified experimental environment and in a more complex, natural one. Among these factors, the low density of parasitoids in natural populations is such that the interference observed in experimental conditions (Fauvergue et al. 2006; C.T., personal observation) must be low. This low interference should lead to stronger parasitoid aggregation on the best patches. The other factor that may drive natural populations away from an ideal free distribution is the risk of hyperparasitism. Larvae of aphid parasitoids are themselves hosts of hyperparasitoids, most of which are solitary, that is, a single hyperparasitoid develops in a parasitoid larva. The patch use behaviour of hyperparasitoids depends on the number of primary parasitoids in the patches, so their presence in the environment is expected to select for strategies of habitat choice by parasitoids associated with risk spreading rather than the maximization of hosts parasitized (Rosenheim 1998; Brodeur \& Rosenheim 2000).

\section{Methods}

\section{Sample collection}

On the 8th of June 2004, we collected patches of Aphis nerii in a continuous hedge of oleander, Nerium oleander, located in Antibes, southeastern France $\left(43^{\circ} 34^{\prime} \mathrm{N}, 7^{\circ} 05^{\prime} \mathrm{E}\right)$. Twenty plants were sampled, $5 \mathrm{~m}$ apart from each other, and 10 aphid colonies were collected on each plant. Because we 
wanted to test a model predicting the distribution and fitness of parasitoids as a function of aphid densities, we collected patches containing at least one mummified aphid, and giving a wide range of aphid densities. Aphis nerii typically forms very dense colonies on young shoots of $N$. oleander, covering the apex and the 3-6 youngest leaves of the shoot. Each shoot was cut under the lowest aphid of the colony and put in a plastic bag before being transported to the laboratory. For each patch, aphids and mummies were counted, and each mummy was individually isolated in a glass vial. Isolated mummies were checked daily during 1 month, and emerged parasitoids were identified as either Lysiphlebus testaceipes, other primary parasitoids, or hyperparasitoids (a fourth possibility being that nothing emerged from the mummy). Emerging $L$. testaceipes were sexed and put in $70 \%$ ethanol until DNA extraction.

\section{Laboratory procedures}

Individual genomic DNA was extracted as described in Estoup et al. (1996), using $160 \mu \mathrm{L}$ of $10 \%$ Chelex-100 suspension and $9 \mu \mathrm{L}$ of proteinase $\mathrm{K}(10 \mathrm{mg} / \mathrm{mL})$. Individual genotypes were determined at 10 microsatellite loci (Lysi1b6, Lysi5a12, Lysi5c4, Lysi5e1, Lysi6b12, Lysi6f4, LysiC1158, LysiC5158, LysiF10, LysiH02; described in Fauvergue et al. 2005). Fluorescent polymerase chain reactions (PCR) were carried out using the QIAGEN multiplex PCR kit. Two reactions were performed for each individual, one with Lysi5c4 and LysiC5158 primer pairs, the other one with the eight other primer pairs. Both duplex and octoplex reactions started with a 15-min step of Taq polymerase activation at $95^{\circ} \mathrm{C}$, and ended with an elongation step of $30 \mathrm{~min}$ at $60^{\circ} \mathrm{C}$. For the duplex reaction, the activation step was followed by 30 cycles of denaturation for $30 \mathrm{~s}$ at $94^{\circ} \mathrm{C}$, hybridization for $1 \mathrm{~min}$ at $56^{\circ} \mathrm{C}$, and polymerization for $1 \mathrm{~min}$ at $72{ }^{\circ} \mathrm{C}$. For the octoplex reaction, there were 24 cycles and hybridization temperature was $52{ }^{\circ} \mathrm{C}$. Allele sizes were estimated on an automated sequencer ABI PRISM 3100 (Applied Biosystems) and read with STRAND software version 2.2.200 (Toonen \& Hughes 2001).

\section{Statistical analysis}

We conducted a parentage analysis to estimate the number of clutches and the number of individuals of each clutch (clutch size) in each sampled patch. Parentage analysis was carried out with COLONY software version 1.2 (Wang 2004). This software uses likelihood methods to reconstruct full-sib and half-sib families for haplodiploid species, without prior knowledge on the parental genotypes. Lysiphlebus testaceipes males are attracted to virgin females exclusively, so females have a low probability of multiple mating (Fauvergue et al. in press). However, males possibly mate with several females. We thus assumed single mating for females and multiple mating for males, and looked for full-sib families within paternal half-sib families. Without prior knowledge of the rate of allelic dropouts or other sources of typing errors, a realistic error rate of 0.05 was assumed for all loci. Having inferred the clutch to which each sampled individual in each patch belonged, we were able to estimate the number and the size of clutches laid in each patch of the habitat. These were the variables further analysed in the test of the IFD.

Predictions of the ideal free distribution were tested by means of generalized linear models (GLM), using the GENMOD procedure in the SAS statistical package version 9.1 (SAS Institute Inc. 1999). The IFD predicts that at equilibrium, the distribution of individual fitness among patches is independent of the quantity of resource and the number of competitors in each patch. Thus, we used the estimated size of each clutch in each patch as the dependent variable, and the number of hosts and the estimated number of clutches in each patch as explanatory variables. Because the actual number of hosts at the time parasitoids exploited the patches (about 1 week before aphid mummification) cannot exactly be known, we used the sum of living and mummified aphids as an indicator of it. Mummified aphids necessarily had to be on the patch at the time of exploitation, and aphids living at the time of sampling are the ones that escaped parasitism plus their offspring, so it is directly linked to the number of aphids living at the time of exploitation. If the L. testaceipes population is at the IFD, neither the number of aphids in the patch nor the number of clutches in the patch should have a significant effect on clutch size. The numbers of hosts and clutches per patch were not normally distributed, thus the natural logarithm of both explanatory variables was used in order to limit the weight of extremely high values in the adjustment of the GLM. Family size is a count data that violate the assumptions of normality and homoscedasticity. We therefore implemented the model with a Poisson distribution and a log-link function. The IFD also predicts that the number of parasitoid clutches in a patch should increase with the number of hosts per patch. To estimate the aggregation of parasitoids, we used a GLM with the number of clutches in a patch as a dependent variable, and the number of aphids in the patch as an explanatory variable. Again, a Poisson distribution and a $\log$-link function were used. Finally, to investigate whether hyperparasitism affects the distribution and fitness of female L. testaceipes, the rate of hyperparasitism, computed as the ratio of the number of hyperparasitoids found in a patch on the number of mummies in the patch, was introduced as a covariate in both GLMs. A logistic regression was used to test the effects of the number of aphids and the number of mummies on the rate of hyperparasitism. 
Table 1 Mean, minimum and maximum values for the variables used in the test of the ideal free distribution, computed on the 168 patches for which Lysiphlebus testaceipes genotypes were available

\begin{tabular}{lrrr}
\hline Variable & Mean & Min. & Max. \\
\hline Aphids per patch & 568.3 & 25 & 3836 \\
Mummies per patch & 16.6 & 1 & 178 \\
Emerging L. testaceipes per patch & 4.9 & 1 & 42 \\
Emerging hyperparasitoids per patch & 2.5 & 0 & 56 \\
Estimated total number of families & 111 & - & - \\
Estimated number of clutches per patch & 2.7 & 1 & 9 \\
Estimated clutch size per patch & 1.8 & 1 & 15 \\
\hline
\end{tabular}

\section{Results}

Our sampling strategy allowed collecting a wide range of patch densities, both in terms of living aphids and mummies (Table 1). Primary parasitoids that emerged from the collected mummies were almost exclusively Lysiphlebus testaceipes, with rare exceptions being Lysiphlebus fabarum and Trioxys sp. Hyperparasitoids included wasps from the superfamilies Cynipoidea such as Alloxysta sp., and Chalcidoidea such as Pachyneuron sp. Because all primary parasitoid and hyperparasitoid species found were solitary, what emerged from each of the 3076 collected mummies was either one $L$. testaceipes ( 869 cases, $28 \%$ ), one primary parasitoid of another species ( 30 cases, $1 \%$ ), one hyperparasitoid (468 cases, $15 \%$ ) or nothing (1739 cases of larval mortality, $56 \%$ ).

The genetic variability of the parasitoid population was rather low (Table 2; mean heterozygosity $=0.319$ ). Locus LysiF10 was not used in the parentage analysis because it was monomorphic. The locus Lysi5158 was also excluded because it produced both stutters and artefact patterns, which made it unreadable. The number of alleles of used loci ranged from two to seven (median $=4$ ), with a highly skewed distribution for loci Lysi1158, Lysi5c4 and Lysi5e1, for which a single allele had a frequency higher than 0.9. The COLONY software assumes Hardy-Weinberg equilib- rium and no linkage disequilibrium between loci. These assumptions could not be tested with our sample because it explicitly included sibs, which systematically biases genotype distribution. However, Fauvergue et al. (2005) could not reject Hardy-Weinberg and linkage equilibria (except between Lysi6b12 and LysiH02) in an independent sample from the same population. Of the 869 genotyped $L$. testaceipes individuals, 818 individuals, distributed among 168 patches, gave interpretable genotypic patterns.

The observed distribution differed from the IFD. Although clutch size in each patch was independent on the number of hosts $\left(n=453\right.$; log-likelihood ratio test: $\chi^{2}=2.62$; $P=0.106$; Fig. 1b), it depended on the number of clutches per patch, which reflects the number of foundress females which had exploited the patch $\left(n=453 ; \chi^{2}=65.79\right.$; $P<0.0001)$. Surprisingly, clutch size increased with the number of clutches in the patch (associated slope in the GLM with log-link function = 1.0746; Fig. 1a). In qualitative accordance with the IFD prediction, the number of clutches in the patches increased with the number of hosts in the patches $\left(n=167 ; \chi^{2}=14.71 ; P=0.0001 ;\right.$ Fig. 2$)$. Nevertheless, the average clutch size was very low with respect to the number of available hosts, most of the clutches having only one member (Table 1; Fig. 1a).

The rate of hyperparasitism (number of hyperparasitoids emerged/number of mummies) in the patches was relatively high (mean: $11.2 \%$; $95 \%$ confidence limits to the mean: 9.2-13.3). It was positively related to the number of mummies $\left(n=168 ; \chi^{2}=189.86 ; P<0.0001\right)$, but not to the number of aphids in the patches $\left(n=168 ; \chi^{2}=0.83\right.$; $P=0.3623)$. The rate of hyperparasitism affected neither the number of families in the patches $\left(n=168 ; \chi^{2}=0.01\right.$; $P=0.9427)$, nor the size of families in the patches $(n=168$; $\chi^{2}=0.11 ; P=0.738$ ).

\section{Evaluation of the statistical method}

In this study, parentage relationships inferred from molecular data were used to test the IFD predictions. Yet, the microsatellite markers used were not very variable

Table 2 Allelic frequencies and observed heterozygosity at the eight Lysiphlebus testaceipes microsatellite loci for the population used in the study

\begin{tabular}{llllllll}
\hline Locus ID & Allele 1 & Allele 2 & Allele 3 & Allele 4 & Allele 5 & Allele 6 & Allele 7 \\
\hline Lysi1158 & 0.91 & 0.09 & & & & 0.096 \\
Lysi1b6 & 0.565 & 0.417 & 0.018 & & & & 0.358 \\
Lysi5a12 & 0.534 & 0.401 & 0.043 & 0.009 & 0.009 & 0.004 & 0.436 \\
Lysi5c4 & 0.892 & 0.06 & 0.024 & 0.024 & & & 0.008 \\
Lysi5e1 & 0.991 & 0.009 & & & & 0.036 \\
Lysi6b12 & 0.78 & 0.088 & 0.074 & 0.018 & 0.018 & 0.013 & 0.009 \\
Lysi6f4 & 0.513 & 0.404 & 0.078 & 0.005 & & 0.559 \\
LysiH02 & 0.461 & 0.304 & 0.161 & 0.035 & 0.022 & 0.017 & 0.393 \\
\hline
\end{tabular}



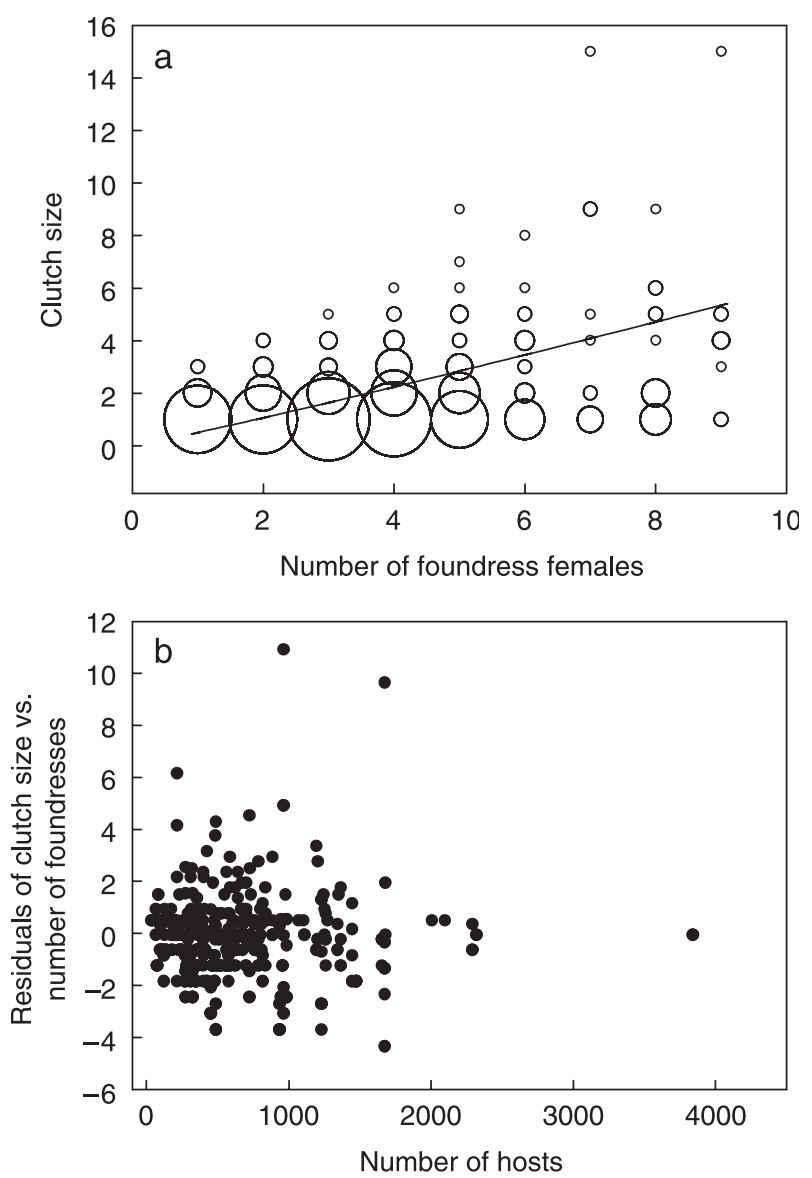

Fig. 1 Effect of the number of foundress females and the number of hosts per patch on clutch size. Clutch size and number of foundresses are estimated by parentage analysis, and the number of hosts is directly counted as the sum of living and mummified aphids. (a) Observed (bubbles) and predicted (line) clutch size vs. number of foundresses per patch. Predicted values are given by the most parsimonious GLM: clutch size $=\exp [-0.6841+$ $1.0746 \times \log$ (number of foundresses)] (see text for details). Bubble area is proportional to the number of clutches. (b) Residuals of the above mentioned model vs. number of hosts per patch. Each dot corresponds to a clutch in a patch $(n=453)$.

(Table 2). The goal of the evaluation of the method was therefore to check if the low variability of the markers could have caused systematic biases in our inferences on the parasitoids' distribution and reproduction. Particularly, we tested whether the two main results, low individual fitness and positive density-dependent fitness, were artefacts of the analysis. To do this, we used a demo-genetic model that simulates a panmictic population of parasitoids with the same genetic variability as the one observed in the actual population (Table 2). This population was distributed across patches either randomly or according to the IFD (see Appendix for details), and produced as many offspring as were genotyped in the actual population. Parentage

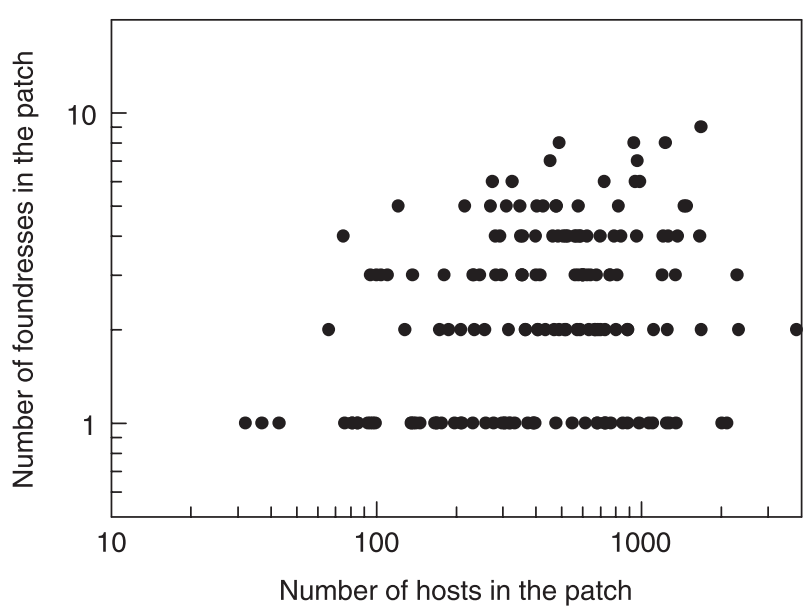

Fig. 2 Number of foundress females per patch vs. number of hosts per patch. The number of foundresses is estimated by parentage analysis, and the number of hosts is directly counted as the sum of living and mummified aphids. Each dot corresponds to a patch $(n=168)$.

analyses were then performed on simulated populations. To quantify the performance of the analysis in regard to the possible underestimation of family size, we computed for each simulation the number of full sib pairs that were erroneously assigned unrelated as well as the number of unrelated pairs that were erroneously assigned full sibs. The combination of these two kinds of error gives the tendency of the analysis to over- or underestimate family size. In addition, we computed for each simulation the ratio of average inferred family size on average actual family size. We then evaluated our capacity to test predictions of the IFD by applying the GLMs mentioned above to the simulated data. We tested the sensitivity of the analysis to the number of parasitoid foundresses in the population, because increasing the size of the parental population for a given level of genetic variability increases the number of foundresses which could bear similar genotypes, thereby possibly affecting the performance of the parentage analysis.

Errors in parentage analysis led to both the splitting of families and the grouping of unrelated individuals, but the latter type of error was more frequent (Fig. 3a). This resulted in the overestimation of family size, especially when many foundresses were simulated (Fig. 3b), that is, when actual family size was low (the total number of offspring was fixed, so increasing the number of foundresses decreased the number of offspring per foundress). Hence, assignment errors resulted in a relatively inaccurate reconstruction of families but not in a systematic bias towards small families, so the very low offspring production observed in the field population (Table 2; Fig. 1) was probably not an artefact due to inaccurate parentage analysis. 

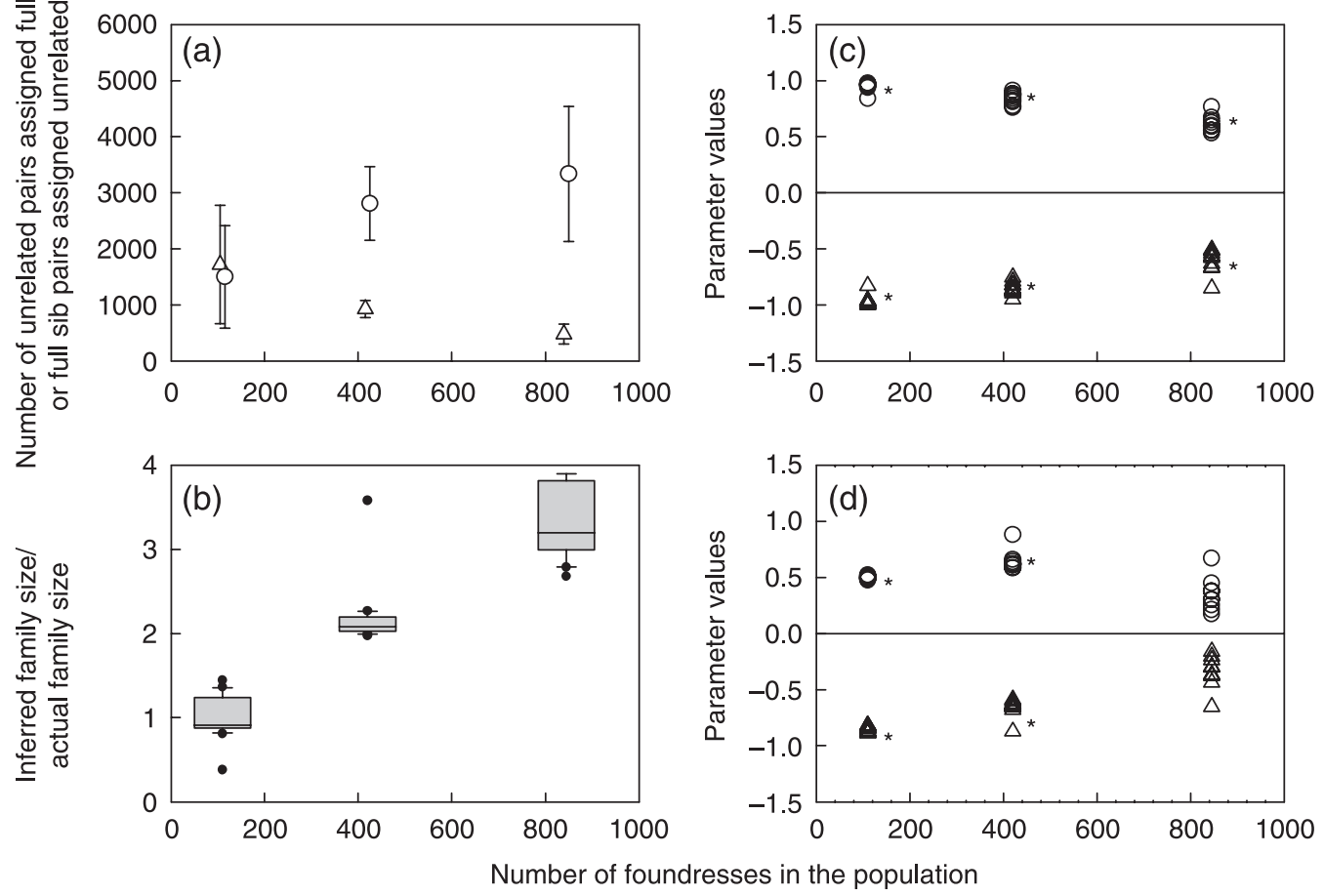

Fig. 3 Evaluation of the method of parentage analysis for the test of ideal free distribution, as a function of the number of mothers in the population. (a) Number of unrelated pairs that were assigned full sibs (circles) and number of full sib pairs that were assigned unrelated (triangles), grouped for both random and ideal free distributions (results are identical for both conditions). (b) Ratio of the average size of families inferred by parentage analysis on the average size of families actually simulated, grouped for both random and ideal free distributions (results are identical for both conditions). (c,d) Parameter estimates of the GLMs for the effects of number of hosts in the patch (circles) and number of foundresses in the patch (triangles) on clutch size in the patch. In c, random distribution was simulated, and in $\mathrm{d}$, ideal free distribution was simulated. Parameter estimates are shown for each of the 10 replicates performed for every condition. Asterisks indicate parameter estimates that differed significantly from zero for each replicate (no asterisk indicates that the parameter was never significantly different from zero).

The GLM statistical analyses on the simulated data showed that in all random distributions, both the number of hosts and number of mothers affected individual fitness in the expected direction: fitness increased with increasing number of hosts and decreasing number of foundresses (Fig. 3c). In simulated ideal free distributions, fitness also increased with increasing number of hosts and decreasing number of mothers, except when many foundresses were in the population (Fig. 3d). These effects are thought to arise from assignment errors, which tend to randomize the assignment of offspring among families. This leads to a random distribution of offspring even if the mothers actually followed an IFD. The better fit to IFD with many mothers in the population can seem surprising, since this was the case when the parentage analysis performed the worst (Fig. 3a). However, in this case, foundresses are so many that they produce very few offspring per patch. Because the test of the IFD concerns fitness per patch, it is less sensitive to assignment errors when clutches are very small and very few individuals emerging from the same patch are related to each other. Importantly, in no occasion did family size increase with the number of mothers in a patch. Thus, the positive density dependence observed in the field is not an artefact due to errors in parentage analysis.

The evaluation of the statistical method indicates that the parentage analysis was not powerful enough to discriminate ideal free from random distribution, except when foundresses produced very few offspring per patch. This is clearly due to the low variability of the genetic markers used. Unfortunately, such a low variability can hardly be compensated by increasing the number of loci to enhance the power of parentage analysis (Bernatchez \& Duchesne 2000). Despite the low power of the statistical method, and importantly for the following discussion, our evaluation shows that the two main results discussed below, small clutch size and positive density-dependent clutch size, were not artefact because of the quite poor performance of the parentage analysis. 


\section{Discussion}

The aim of this study was to characterize the distribution of parasitoids among aphid patches in the field. Particularly, we tested whether individual fitness on a host patch was independent of the number of hosts and the number of parasitoids having exploited the patch, and whether the number of parasitoids exploiting a patch increased with the number of hosts in the patch. For this, the number of parasitoid females having exploited a patch and their respective fitness were estimated via a parentage analysis on their offspring. Our results indicate that the population studied did not follow an ideal free distribution. The number of parasitoid females which reproduced on a patch admittedly increased with the number of hosts in the patch (Fig. 2), as predicted by the IFD. However, although the number of offspring produced per female was independent of the number of hosts, it was affected by the number of females which had reproduced on the patch. Surprisingly, with respect to the general assumption that increasing number of individuals increases competition and therefore reduces fitness gain, individual fitness increased with the number of females that had reproduced on the patch (Fig. 1).

The first remarkable result of this study is the very low individual fitness per patch. Several non-exclusive hypotheses can explain the low individual fitness per patch. First, the low proportion of mummies from which Lysiphlebus testaceipes adults emerged (869 L. testaceipes out of 3076 mummies) shows that larval mortality strongly decreased individual fitness. Causes of larval mortality were hyperparasitism, predation, and dented mummies resulting from either natural causes or our manipulation. However, even if all the parasitoids had emerged from the mummies, the average family size would have been quite low in regard to the number of hosts available. The second hypothesis involves the dynamical dimension of the distribution of parasitoids among patches, with the possibility that initially randomly distributed foundresses would progressively redistribute according to the IFD. In this case, genetic data on offspring would not provide a snapshot of the possible final IFD, but rather a track of the whole convergence process, during which parasitoids rapidly switch patches (as observed by Fauvergue et al. 2006) after having laid few eggs in each. Hence, the low offspring production per patch would be the result of the redistribution of the parasitoids during the phase of convergence towards the IFD. The third hypothesis involves risk-spreading behaviour by the parasitoids. In the population of L. testaceipes, as well as in other populations studied in the field (Müller \& Godfray 1998; Van Veen et al. 2002; Mackauer \& Völkl 2005), the rate of hyperparasitism was high and positively related to the number of mummies per patch. In such a situation, laying only few eggs per patch is adaptive because it maximizes the rate of offspring production (Ayal \& Green 1993), unlike when the risk of clutch destruction is uniform (Hopper et al. 2003). If the parasitoids have no information on the actual abundance of hyperparasitoids during their offspring's development, a fixed risk-spreading strategy should be selected for, and should have been directly observed in the parasitoids' behaviour, at least in the population we studied. Yet, this hypothesis contrasts with previous observation of the parasitoid's high rate of reproduction on host patches. In their test of an IFD model on L. testaceipes in greenhouse, Fauvergue et al. (2006) report reproduction rates of about 25 offspring per hour of patch exploitation. In the same population than the one studied here, mated L. testaceipes females attacked 5.1 Aphis nerii per minute, resulting in up to 220 attacks on a colony bearing 150 aphids (Fauvergue et al. in press). A last and more satisfying hypothesis which can explain the low fitness of parasitoids per patch involves the interaction between parasitoids and aphid-tending ants. In the population studied, foraging $L$. testaceipes were aggressively chased by Argentine ants, Linepithema humile, which were excluded from the set-up in the two previous studies. This is very likely to shorten the time available to parasitoids to lay their eggs and ultimately lower the number of offspring produced in each colony (Völkl \& Mackauer 1993).

The other striking result of this study is the positive relation between the number of foundresses per patch and individual fitness. The positive effect of the number of parasitoids on individual fitness challenges seriously the assumption of competition-driven negative density dependence which underlies the ideal free distribution paradigm. By definition, competition is the decrease in individual fitness due to the presence of other individuals, and occurs when the resource is the factor limiting fitness gain. In both theoretical models and empirical studies of the IFD, this limitation by resource has been often forced by imposing unrealistically high ratios of foragers to resource (e.g. simulation models: Harley 1981, 30 predators in two patches; Bernstein et al. 1988, 900 predators in 49 patches; Ranta et al. 1999, 10000 predators in 1000 patches; empirical tests: Harper 1982, 24 ducks in two patches; Pusenius \& Schmidt 2002, 60-140 voles in four patches). However in the wild, population densities may not be as high as in experimental set-ups or models, and the strong competition created artificially may be an exception rather than the rule (Greene \& Stamps 2001). Especially for most parasitoid species, where it is rare to see more than one individual per patch at the same time (Cronin 2003; Wertheim et al. 2003), models of habitat selection based solely on competition (and all the more, those based on interference) may be inappropriate. Three mechanisms could underlie the positive relationship between the number of foundresses and individual fitness. First, the larval survival of offspring may be higher when many individuals reproduce on the 
patch. This could be caused for instance by the dilution of hyperparasitism or predation on offspring. However, the positive relation between the number of mummies on a patch and the rate of hyperparasitism found in this and other studies (Chow 2000; but see Schooler et al. 1996; Müller \& Godfray 1998; Van Veen et al. 2002) indicates that dilution of hyperparasitism does not occur. Second, individuals may take the decision to lay more eggs on patches where other individuals have already reproduced. This could be adaptive in the above-mentioned case of hyperparasitism dilution, but also if restricted dispersal constrains the mating opportunities for offspring to the local patch. Third, the number of foundresses and clutch size could be both correlated to a third, confounding, variable. Our data do not allow to disentangle these hypotheses, and further behavioural studies could shed light on the actual mechanism causing the observed positive density dependence.

Negative density dependence has been a keystone concept for theoreticians to explain stability in predator-prey and parasite-host systems (Bernstein 2000). Results like ours participate to the current trend of documenting positive density dependence and the ensuing instability of natural systems. The interplay between positive and negative density dependence in habitat selection was initially acknowledged by Fretwell \& Lucas (1970) but largely overlooked in further theoretical developments. Recent theoretical work shows that habitat selection by 'ideal free' organisms experiencing positive density dependence can result in the aggregation of individuals regardless of differences in habitat quality (Greene \& Stamps 2001) and lower extinction thresholds in metapopulations (Greene 2003). Accordingly, a cost/benefit approach on attraction to conspecifics shows how the positive effect of conspecifics density on fitness can outweigh competition to lead to adaptive aggregation of individuals (Donahue 2006). Given the widespread occurrence of positive density dependence (Berec et al. 2007), the consideration of other factors than competition seems to be the key for understanding habitat selection and patterns of distribution for a variety of biological systems. Our results suggest that predation and parasitism on offspring might be such factors.

\section{Conclusion}

Using parentage analyses to infer individual fitness of parasitoid females in the field, this study reveals low offspring production per patch and a positive relation between density and fitness. This suggests that intraspecific competition is not the main factor driving population distribution, and that intraguild interactions may predominate. At the methodological level, the parentage analysis was not perfectly reliable but powerful enough to reveal the main features of parasitoids' distribution discussed above. Such method could be fruitful to explore habitat selection in other organisms in which genetic variability is high enough.

\section{Acknowledgements}

We thank all members of the team BPI at INRA Sophia-Antipolis, for the time spent sorting mummies in the laboratory, Réjane Streiff for advice on microsatellite analysis, Joseph Tentelier for crucial insights into the process of breeding dispersal, Jinliang Wang for modifying the output format of COLONY, thereby facilitating further data processing, and two anonymous referees for their helpful comments on the manuscript. This study was financed through grant \# 2002-12324 from 'INRA Santé des Plantes et Environnement - Région PACA' allocated to X.F. and C.T., a grant from the French Academy of Agriculture allocated to C.T., and a grant from 'INRA Santé des Plantes et Environnement' allocated to X.F.

\section{References}

Ayal Y, Green RF (1993) Optimal egg distribution among host patches for parasitoids subject to attack by hyperparasitoids. American Naturalist, 141, 120-138.

Beckmann JP, Berger J (2003) Using black bears to test ideal-free distribution models experimentally. Journal of Mammalogy, 84, 594-606.

Berec L, Angulo E, Courchamp F (2007) Multiple Allee effects and population management. Trends in Ecology E Evolution, 22, 185-191.

Bernatchez L, Duchesne P (2000) Individual-based genotype analysis in studies of parentage and population assignment: how many loci, how many alleles? Canadian Journal of Fisheries and Aquatic Sciences, 57, 1.

Bernstein C (2000) Host-parasitoid models: the story of a successful failure. In: Parasitoid Population Biology (eds Hochberg ME, Ives AR), pp. 41-57. Princeton University Press, Princeton, New Jersey.

Bernstein C, Kacelnik A, Krebs JR (1988) Individual decisions and the distribution of predators in a patchy environment Journal of Animal Ecology, 57, 1007-1026.

Berry O, Tocher MD, Sarre SD (2004) Can assignment tests measure dispersal? Molecular Ecology, 13, 551-561.

Brodeur J, Rosenheim JA (2000) Intraguild interactions in aphid parasitoids. Entomologia Experimentalis et Applicata, 97, 93-108.

Carlsson J, Carlsson JEL, Olsen KH et al. (2004) Kin-biased distribution in brown trout: an effect of redd location or kin recognition? Heredity, 92, 53-60.

Castric V, Bernatchez L (2004) Individual assignment test reveals differential restriction to dispersal between two salmonids despite no increase of genetic differences with distance. Molecular Ecology, 13, 1299-1312.

Cezilly F, Benhamou S (1996) Optimal foraging strategies: a review. Revue D Ecologie-la Terre et la Vie, 51, 43-86.

Chow A (2000) Influence of host distribution on foraging behaviour in the hyperparasitoid wasp, Dendrocerus carpenteri. Entomologia Experimentalis et Applicata, 97, 57-66.

Cronin JT (2003) Patch structure, oviposition behavior, and the distribution of parasitism risk. Ecological Monographs, 73, 283-300. 
Donahue MJ (2006) Allee effects and conspecific cueing jointly lead to conspecific attraction. Oecologia, 149, 33-43.

Estoup A, Largiadèr CR, Perrot E, Chourrout D (1996) Rapid one-tube extraction for reliable PCR detection of fish polymorphic markers and transgenes. Molecular Marine Biology and Biotechnology, 5, 295-298.

Fauvergue X, Boll R, Lapchin L et al. (2006) Habitat assessment by parasitoids: consequences for population distribution. Behavioral Ecology, 17, 522-531.

Fauvergue X, Lo Genco A, Lo Pinto M (in press) Virgins in the wild: mating status affects the behaviour of a parasitoid foraging in the field. Oecologia

Fauvergue X, Tentelier C, Genson G, Audiot P, Guillemaud T, Streiff R (2005) Microsatellite DNA markers for Lysiphlegbus testaceipes. Molecular Ecology Notes, 5, 109-111.

Fretwell SD, Lucas HL (1970) On territorial behavior and other factors influencing habitat distribution in birds. Acta Biotheoretica, 19, 16-36.

Girman DJ, Mills MGL, Geffen E, Wayne RK (1997) A molecular genetic analysis of social structure, dispersal, and interpack relationships of the African wild dog (Lycaon pictus). Behavioral Ecology and Sociobiology, 40, 187-198.

Greene CM (2003) Habitat selection reduces extinction of populations subject to Allee effects. Theoretical Population Biology, 64, $1-10$.

Greene CM, Stamps JA (2001) Habitat selection at low population densities. Ecology, 82, 2091-2100.

Harley CB (1981) Learning the evolutionarily stable strategy. Journal of Theoretical Biology, 89, 611-633.

Harper DG (1982) Competitive foraging in mallards: 'ideal free' ducks. Animal Behaviour, 30, 575-584.

Hopper KR, Rosenheim JA, Prout T, Oppenheim SJ (2003) Within-generation bet hedging: a seductive explanation? Oikos, 101, 219-222.

Kennedy M, Gray RD (1993) Can ecological theory predict the distribution of foraging animals? A critical analysis of experiments on the ideal free distribution. Oikos, 68, 158-166.

Mackauer M, Völkl W (2005) Sex ratio shift caused by hyperparasitism in the solitary parasitoid Lysiphlebus hirticornis (Hymenoptera: Braconidae: Aphidiinae). European Journal of Entomology, 102, 475-481.

Moyer MA, McCown JW, Eason TH, Oli MK (2006) Does genetic relatedness influence space use pattern? A test on Florida black bears. Journal of Mammalogy, 87, 255-261.

Müller CB, Godfray HCJ (1998) The response of aphid secondary parasitoids to different patch densities of their host. Biocontrology, 43, 129-139.

Pusenius J, Schmidt KA (2002) The effects of habitat manipulation on population distribution and foraging behavior in meadow voles. Oikos, 98, 251-262.

Rabasse JM, Wyatt IJ (1985) Biology of aphids and their parasites in greenhouses. In: Biological Pest Control - The glasshouse experience (eds Hussey NW, Scopes NEA), pp. 66-73. Blandford Press, Poole, UK.

Ranta E, Lundberg P, Kaitala V (1999) Resource matching with limited knowledge. Oikos, 86, 383-385.
Rosenheim JA (1998) Higher -order predators and the regulation of insect herbivore populations. Annual Review of Entomology, 43, 421-447.

Rousset F (1997) Genetic differentiation and estimation of gene flow from $F$-statistics under isolation by distance. Genetics, 145, 1219-1228.

SAS Institute Inc. (1999) SAS/Stat User's Guide, Version 8. SAS Institute, Cary, North Carolina.

Schooler SS, Ives AR, Harmon J (1996) Hyperparasitoid aggregation in response to variation in Aphidius ervi host density at three spatial scales. Ecological Entomology, 21, 249-258.

Sera WE, Gaines MS (1994) The effect of relatedness on spacing behavior and fitness of female prairie voles. Ecology, 75, 15601566.

Sutherland WJ (1983) Aggregation and the ideal free distribution. Journal of Animal Ecology, 52, 821-828.

Tentelier C, Fauvergue X (2007) Herbivore-induced plant volatiles as cues for habitat assessment by a foraging parasitoid. Journal of Animal Ecology, 76, 1-8.

Tentelier C, Wajnberg E, Fauvergue X (2005) Parasitoids use herbivore-induced information to adapt patch exploitation behaviour. Ecological Entomology, 30, 739-744.

Tentelier C, Desouhant E, Fauvergue X (2006) Habitat assessment by parasitoids: mechanisms for patch use behavior. Behavioral Ecology, 17, 515-521.

Toonen RJ, Hughes S (2001) Increased throughput for fragment analysis on an ABI PRISM 377 automated sequencer using a membrane comb and STRAND software. BioTechniques, 31, 1320-1324.

Tregenza T (1995) Building on the ideal free distribution. Advances in Ecological Research, 26, 253-307.

Van Veen FJF, Muller CB, Adriaanse ICT, Godfray HCJ (2002) Spatial heterogeneity in risk of secondary parasitism in a natural population of an aphid parasitoid. Journal of Animal Ecology, 71, 463-469.

Völkl W, Mackauer M (1993) Interactions between ants attending Aphis fabae ssp. cirsiiacanthoidis on thistles and foraging parasitoid wasps. Journal of Insect Behavior, 6, 301-312.

Wahlstrom LK, Kjellander F (1995) Ideal free distribution and natal dispersal in female roe deer. Oecologia, 103, 302-308.

Wang J (2004) Sibship reconstruction from genetic data with typing errors. Genetics, 166, 1963-1979.

Wertheim B, Vet LEM, Dicke M (2003) Increased risk of parasitism as ecological costs of using aggregation pheromones: laboratory and field study of Drosophila-Leptopilina interaction. Oikos, 100, 269-282. 
Appendix Simulation model used to estimate the performance of parentage analysis for testing IFD predictions

\section{Initial conditions: habitat and parental genotypes}

In every simulation, the habitat consisted of 168 host patches, among which hosts were distributed as observed in the field (see Table 2). At the beginning of each simulation run, a panmictic parental population of parasitoids was created as follows. A variable number of females (see below, Sensitivity analysis) were assigned three alleles for each of the eight microsatellite loci. The first two alleles corresponded to the female's diploid genotype, and the third allele corresponded to the haploid genotype of the male to which the female was singly mated. Each allele was drawn randomly from the allelic frequency distribution observed in the field population (Table 1).

\section{Distribution of foundresses across patches}

Foundresses were distributed simultaneously among the 168 patches. To simulate ideal free distribution, we used Sutherland's (1983) interference model:

$P_{i}=k H_{i}^{1 / m}$

where $P_{i}$ and $H_{i}$ are the number of parasitoid foundresses and hosts on patch $i, k$ is a normalizing constant so that the sum of foundresses across patches equals the total foundress population, i.e. $k=\sum_{i} P_{i} / \sum_{i} H_{i}^{1 / m}$, and $m$ is the degree of interference between parasitoids (experimentally estimated to be 1 for Lysiphlebus testaceipes; Fauvergue et al. 2006).

To simulate a random distribution, the number of foundresses reproducing on each patch was drawn from a Poisson distribution parameterized with the ratio of the total number of foundresses in the population to the number of patches $(=168)$ in the habitat. For both ideal free and random distribution, the individual foundresses placed in each patch were drawn randomly from the initial pool of females.

\section{Parasitoid reproduction}

The number of offspring produced by each foundress $j$ on each patch $i$ was computed using a type I functional response:

$w_{i j}=Q \times H_{i} \times P_{i}^{-m}$

where $Q$ is the individual efficiency of parasitism, that is, $Q=\Sigma_{i} \Sigma_{j} w_{i j} / \Sigma_{i} H_{i}$, and $m$ is again interference, set to 1 .

For each offspring, the probability of being a male was 0.38 (= sex ratio observed in the actual population), and parental alleles were inherited following Mendelian rules for haplodiploids. The total number of offspring produced $\left(\Sigma_{i} \Sigma_{j} w_{i j}\right)$ was set to 845 , to match the number of individuals genotyped in the actual population.

\section{Sensitivity analysis}

We analysed the sensitivity of the model to the number of foundresses in the population. It could affect parentage analysis because it affects the number of foundresses which could bear similar genotypes, thereby affecting the probability of grouping unrelated offspring in the same family. The number of foundresses was either 845,420 or 100 . Because the total number of offspring was set to 845 , decreasing the number of foundresses resulted in increasing the average number of offspring produced per foundress $(1,2.01$ and 8.45 , respectively). Ten replicates were performed for each combination of number of foundresses $(845,420$ and 100) and distribution (IFD and random). No typing errors were assumed in the parentage analysis. 